\title{
EL REGISTRO DE EXPLOTACIONES Y SUS POSIBILIDADES EN LA INVESTIGACIÓN GEOGRÁFICA
}

\author{
Juan Cruz Alberdi Collantes \\ Departamento de Geografía de la Universidad del País Vasco \\ Facultad de Filología, Geografía e Historia. Francisco Tomás y Valiente, s/n \\ Apartado 2111.01006 Vitoria-Gasteiz. E-mail: fgpalcoj@vc.ehu.es
}

Resumen: En este artículo analizamos las posibilidades que la incorporación de una nueva fuente de información, los registros de explotaciones, pueden aportar a los estudios de geografía agraria. Esta fuente recoge información relevante sobre los caracteres principales de las explotaciones agrarias, desde datos relativos al titular, cónyuge o personas que colaboran en estas labores, actividades agrarias presentes, régimen de tenencia o utilidad aportada a cada una de las parcelas empleadas con referencias a su localización en el catastro de rústica y sobre fotografía aérea. Para observar sus posibilidades recurrimos al estudio de dos casos prácticos, la tipología de explotación de ovino y los usos del suelo en el caserío vasco. En el primer ejemplo, observamos dos tendencias claramente definidas, que se repiten en todos los sectores productivos representados: una minoritaria, tendente a la especialización y aumento de la capacidad productiva, y la principalmente observada, hacia el abandono de la función agraria. En el segundo, observamos que los aprovechamientos agrarios recaen en un reducido número de explotaciones, coincidentes con aquéllas que han optado por continuar con la actividad.

Palabras clave: caserío, San Sebastián, País Vasco, agricultura, ganadería, usos del suelo, fuentes de información agraria, registros de explotación.

\begin{abstract}
: in this paper we study the possibilities to the farm geography investigation of a new information way, the farm register. This source picks up exploitation main information about the family work, farm activity and production, propriety of the land cover, uses and their relations whit the propriety register and its cartography. In the paper we analyse two practical examples, the typology of sheep exploitation and the use of the land cover in the Basque Country farm exploitation. First, we see two different ways for all production sector: one conducting to the specialisation and increasing productions capacity; and other, more extended, conducting to the abandon of all farm activity. In the second example, we see that the farm use depend of a little farmers number, the cattle who has take the decision of continue with the farm activity.
\end{abstract}

Key words: San Sebastian, Basque Country, agriculture, cattle, land use, farm information sources, farm register. 


\section{Introducción}

Los registros de explotaciones se constituyen en una de las fuentes de información más interesantes en los estudios agrarios y de manera especial en la investigación de realidades espaciales. Recogen datos relativos al número de personas que trabajan en la agricultura, grado de dedicación, actividades de producción e, incluso, base territorial utilizada según usos y régimen de tenencia.

Sin embargo, se trata de una información reciente, con algunas deficiencias en su configuración y con un importante número de unidades productivas que todavía no están registradas. Cuenta con una dificultad añadida: es declaración personalizada y su utilización es de uso restringido, sometida a consultas puntuales y limitadas, y, por el momento, no puede ser tratada estadísticamente, con lo que su utilidad se concreta en la consulta de fichas individuales.

Aun así, al relacionar en un único documento los principales elementos que definen una explotación con indicaciones relativas a su declaración de superficie, en la que se identifican en fotografías aéreas y parcela a parcela superficies utilizadas, usos del suelo y régimen de tenencia de cada explotación, se convierten estos registros en una fuente de investigación de gran valor geográfico.

En este artículo vamos a observar las peculiaridades, posibilidades y deficiencias que los registros de explotaciones presentan a la hora de ser aplicados en el estudio de diferentes características de los espacios agrarios. Para ello, procedemos a desarrollar dos ejemplos a través de las aportaciones obtenidas de estos registros, en primer lugar a partir de su utilización en el estudio de las explotaciones especializadas en una rama productiva, en ovino, y en segundo lugar, a partir de su aplicación a todas las explotaciones que lo han confeccionado en un barrio o área delimitada y controlable a escala humana. Ambos ejemplos se desarrollan en la comarca de San Sebastián.

\section{Qué son los Registros de Explotaciones}

En 1995 se crea esta nueva fuente, en la que se informatizan las características más relevantes de cada una de las explotaciones agrarias de Gipuzkoa ${ }^{1}$. Se renueva anualmente en las oficinas comarcales agrarias y se convierte en obligatoria para optar a cualquier tipo de ayuda o realizar cualquier trámite oficial. Se completa a partir de los datos aportados por el propio agricultor y, por lo general, recoge la información en tres bloques diferenciados: 
a) Personas jurídicas o físicas que trabajan en la explotación: se señalan datos del titular y demás personas que participan en la instalación productiva así como su relación respecto al titular. Edad, dedicación y grado de dedicación son, entre otros, los aspectos reseñados.

b) Actividad agroganadera: se indica la información relativa a las actividades agrícolas de la explotación, especialmente ganadera (tipo de ganado, cantidad, raza, fecha de nacimiento, ...).

c) Declaración de superficie: en este apartado se recogen aspectos relativos al régimen de la propiedad, superficie total labrada e incluso el cultivo en función del suelo utilizado (forrajero, hortaliza, frutal, ...).

En la actualidad, la mayoría de las unidades productivas han formalizado el Registro o lo están haciendo progresivamente, al menos aquellas que ostentan una dedicación a la actividad suficiente como para tener que realizar alguna tramitación oficial o solicitar ayuda pública.

Es una fuente de gran valor para el análisis del espacio agricola y de las unidades agrarias. La información que se recoge está contrastada con la actualización del Catastro de Rústica y localizada sobre el territorio mediante fotografía aérea. La posibilidad de unir producción, productor, superficie utilizada y cultivo permite recoger los caracteres esenciales de una explotación en un único documento.

A pesar de su validez, todavía muestran importantes deficiencias. Su consulta resulta problemática: los datos son de acceso restringido y no existen resúmenes municipales o comarcales sino que se trata de fichas individualizadas que, al menos por el momento, no pueden ser tratadas informáticamente. La información, a su vez, es introducida sin excesivas comprobaciones: en la casilla correspondiente a la ayuda en la explotación, a menudo, sólo se señalan titulares y cotitulares y no hijos u otras personas que puedan trabajar en ella; en algunos casos titularidad y jefatura de la explotación no son correctamente diferenciados; la superficie declarada no siempre es la utilizada y, por lo general, sólo se recoge la propia y la arrendada y, rara vez, la que cede sin ningún acuerdo legal... .

Aun así, esta fuente es la más completa que hemos manejado sobre instalaciones y usos agrícolas. Su empleo aporta las características más relevantes de las explotaciones que expresamente hemos elegido como representativas de los distintos modelos que observamos, permitiendo extrapolar estos datos a los caracteres generales de cada tipología. 


\section{Aplicación al estudio sectorial: importancia del ovino en el caserío vasco}

Analizamos la situación del sector ovino atendiendo a las aportaciones de los registros de explotaciones. Sin embargo, el carácter individualizado y el acceso restringido y puntual de esta información lleva a recurrir a otras fuentes para completar el estudio que proponemos. Esta combinación permite obtener una metodología de aplicación sencilla y con unos resultados interesantes. Las fuentes a las que recurrimos antes de consultar los registros son las siguientes:

- Censo Agrario de 1982, 1989 y 1999. Utilizamos preferentemente los dos primeros para mostrar la evolución que muestra cada sector en ese período.

- Campañas de saneamiento. La Diputación Foral de Gipuzkoa lleva realizando de manera ininterrumpida desde 1987 controles veterinarios del ganado vacuno y ovino. Las aportaciones se ordenan por municipios en dos informes diferenciados, uno para bovino y otro para ovino, y además se recoge el número de cabezas de cada unidad productiva, lo que permite clasificar las ganaderías en función de la cabaña que ostentan ${ }^{2}$.

- Datos de equivalencia entre producciones y necesidades de mano de obra agraria (unidad de trabajo agrario). El Departamento de Industria, Agricultura y Pesca del Gobierno Vasco mediante el decreto 168/1997 del 8 de julio de 1997 regula las unidades mínimas de cultivo en los distintos territorios históricos y comarcas de la C.A.P.V.. La unidad mínima de cultivo requerida para que una explotación tenga consideración de prioritaria es una unidad de trabajo agrícola o U.T.A. El mismo decreto fija el margen bruto de equivalencia de una U.T.A. para cada producción.

A partir de estas fuentes clasificamos y cuantificamos en función de la cabaña ovina las ganaderías que tienen esta actividad en toda la comarca. Tomando una muestra reducida de cada uno de los grupos y a partir de la información que estas explotaciones han declarado en el registro es relativamente sencillo obtener una visión de la situación del sector estudiado. Desarrollamos a continuación uno de estos ejemplos.

\subsection{Aumento generalizado del número de rebaños en los últimos veinte años}

En un área de montaña, con posibilidad de combinar parte de tierras altas con tierras bajas, el ganado lanar encuentra un medio adecuado para su desarrollo. Este sistema se adapta muy bien a la estacionalidad productiva del ovino lechero, con partos centrados de finales de otoño a finales de invierno y con una producción lechera que se prolonga durante los meses de invierno y primavera. El rendimiento máximo se sitúa en los meses de marzo-abril, lo que permite un buen aprovechamiento del pasto de primavera. 


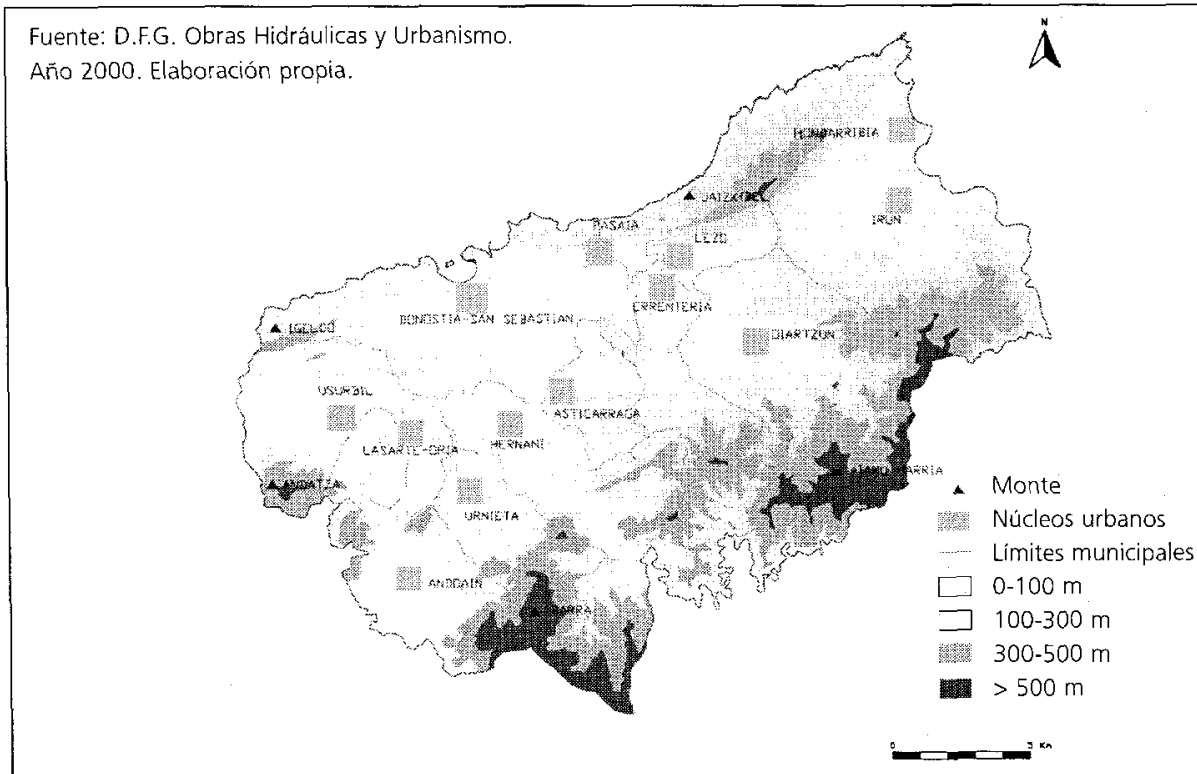

Figura 1. Comarca de Donostialdea-Bidasoa.

La comarca de San Sebastián conserva superficies de pastos de altura que debido al clima húmedo y templado, pueden aprovecharse de manera prolongada ${ }^{3}$. Los montes Adarra, Andatza, Jaizkibel y Aiako Harriak (Figura 1) son sus mejores exponentes, existiendo en todas las poblaciones que limitan con estas estribaciones asociaciones profesionales de ganaderos que se encargan de la ordenación y gestión del suelo de estas zonas ${ }^{4}$.

A pesar de ello, el sector ovino no tiene un considerable desarrollo, especialmente si lo comparamos con el bovino. La asociación de profesionales de ovino en Gipuzkoa, E.L.E. (Asociación de oveja latxa del País Vasco), cuenta en esta comarca con 8 socios, muy lejos de los 89 que formaban parte de la asociación de vacuno de leche (GIFE) o los 70 de la asociación de horticultores de Gipuzkoa (GILBE) ${ }^{5}$. Sin embargo, en 1998 había 490 explotaciones agrarias que poseían este tipo de ganado, tantas como unidades lecheras, de abasto u horticolas ${ }^{6}$.

Mediante los datos publicados en los censos agrarios obtenemos una imagen del sector en la comarca durante la década de los ochenta.

Según el Censo Agrario de 1982 existían en total 276 caseríos que poseían ganado lanar, con una media de 56,4 reses por explotación. En el Censo de 1989 las explotaciones aumentan hasta 391, presentando una media de 45,3 reses por rebaño. Tanto en uno como en otro el número de cabezas por unidad de producción es insuficiente para justificar una dedicación mínima. En función de las necesidades de equivalencia 
en mano de obra se calcula que con 157 ovejas destinadas a ordeño se obtiene una U.T.A., una cifra muy alejada de la media comarcal.

Tabla 1. Número de cabezas de ganado ovino por explotación. Años 1982 y 1989.

\begin{tabular}{|l|ccc|ccc|}
\hline \multirow{2}{*}{ MUNICIPIO } & \multicolumn{3}{c|}{1982} & \multicolumn{4}{c|}{1989} \\
\cline { 2 - 7 } & $\mathrm{N}^{\circ}$ Explot & $\mathrm{N}^{\circ}$ Cab. & Cab/Expl. & $\mathrm{N}^{\circ}$ Explot & $\mathrm{N}^{\circ}$ Cab. & Cab/Expl. \\
\hline ANDOAIN & 27 & 733 & 27,1 & 27 & 1.394 & 51,6 \\
ASTIGARRAGA & & & & 9 & 159 & 17,7 \\
DONOSTIA & 35 & 1.049 & 30,0 & 24 & 1.074 & 44,8 \\
ERRENDERIA & 26 & 1.155 & 44,4 & 26 & 1.114 & 42,8 \\
HERNANI & 42 & 2.056 & 49,0 & 42 & 2.399 & 57,1 \\
HONDARRIBIA & 6 & 318 & 53,0 & 11 & 425 & 38,6 \\
IRUN & 36 & 6.239 & 173,3 & 51 & 2.267 & 44,5 \\
LASARTE-ORIA & & & & 6 & 220 & 36,7 \\
LEZO & 9 & 737 & 81,9 & 8 & 277 & 34,6 \\
OIARTZUN & 37 & 1.708 & 46,2 & 105 & 5.737 & 54,6 \\
PASAIA & 4 & 454 & 113,5 & 4 & 571 & 142,8 \\
URNIETA & 28 & 700 & 25,0 & 37 & 1.173 & 31,7 \\
USURBIL & 26 & 409 & 15,7 & 41 & 897 & 21,9 \\
\hline TOTAL & 276 & 15.558 & 56,4 & 391 & 17.707 & 45,3 \\
\hline
\end{tabular}

Fuente: Censo Agrario 1982 y 1989. Elaboración Propia.

El número de cabezas de ovino que había en Donostialdea-Bidasoa en 1991, 1995 y 1998, así como el número de caserios que presentan este tipo de ganado, queda recogido en el cuadro que exponemos a continuación.

Tabla 2. Ganado ovino. Años 1991, 1995 y 1998.

\begin{tabular}{|c|c|c|c|c|c|c|}
\hline \multirow[b]{2}{*}{ MUNICIPIO } & \multicolumn{2}{|c|}{1991} & \multicolumn{2}{|c|}{1995} & \multicolumn{2}{|c|}{1998} \\
\hline & $\begin{array}{c}\mathrm{N}^{\circ} \\
\mathrm{Cab} .\end{array}$ & $\begin{array}{l}\text { Explot. } \\
\text { Munic. }\end{array}$ & $\begin{array}{c}\mathrm{N}^{\circ} \\
\mathrm{Cab} .\end{array}$ & $\begin{array}{l}\text { Explot. } \\
\text { Munic. }\end{array}$ & $\begin{array}{c}\mathrm{N}^{\circ} \\
\mathrm{Cab} .\end{array}$ & $\begin{array}{l}\text { Explot. } \\
\text { Munic. }\end{array}$ \\
\hline ANDOAIN & 1.927 & 34 & 2.105 & 44 & 2.264 & 47 \\
\hline ASTIGARRAGA & 533 & 13 & 407 & 15 & 456 & 17 \\
\hline DONOSTIA & 1.591 & 33 & 2.232 & 38 & 2.548 & 40 \\
\hline ERRENDERIA & 848 & 10 & 1.203 & 24 & 1.363 & 26 \\
\hline HERNANI & 3.432 & 58 & 3.599 & 59 & 4.139 & 63 \\
\hline HONDARRIBIA & 1.162 & 11 & 1.008 & 14 & 1.214 & 16 \\
\hline IRUN & 2.395 & 53 & 2.831 & 64 & 3.296 & 73 \\
\hline LASARTE-ORIA & 345 & 7 & 364 & 11 & 471 & 10 \\
\hline LEZO & 699 & 6 & 692 & 9 & 952 & 10 \\
\hline OIARTZUN & 6.531 & 99 & 6.966 & 118 & 7.234 & 119 \\
\hline PASAIA & 642 & 4 & 634 & 4 & 377 & 4 \\
\hline URNIETA & 1.168 & 29 & 1.131 & 34 & 1.557 & 40 \\
\hline USURBIL & 1.253 & 45 & 1.476 & 52 & 1.710 & 55 \\
\hline COMARCA & 22.526 & 402 & 24.648 & 486 & 27.581 & 520 \\
\hline
\end{tabular}

Fuente: D.F.G. 1991, 1995 y 1998. Elaboración Propia. 
Constatamos que las reses de ovino aumentan progresivamente, incrementándose en este período de siete años en 5.055 cabezas. En la misma línea, el número de hatos crece, pasando de 402 en 1991 a 520 en 1998. Entre estos años, con ligeras alteraciones, se mantiene el número de cabezas por explotación (56 en 1991 y 53 en 1998). Todos los municipios muestran un proceso similar. Como caso especial cabe recoger el traspaso de Pasaia a Lezo de un rebaño que provoca alteraciones en las cifras de estas poblaciones.

Todo parece indicar que se está dando en ovino un proceso caracterizado por un aumento progresivo del número de explotaciones y de reses mientras la media por explotación se mantiene e incluso disminuye, lo que apunta un descuido en el manejo y provecho de los hatos lanares.

Figura 2. Evolución del número de cabezas de ganado ovino en Gipuzkoa desde1988 hasta1998.

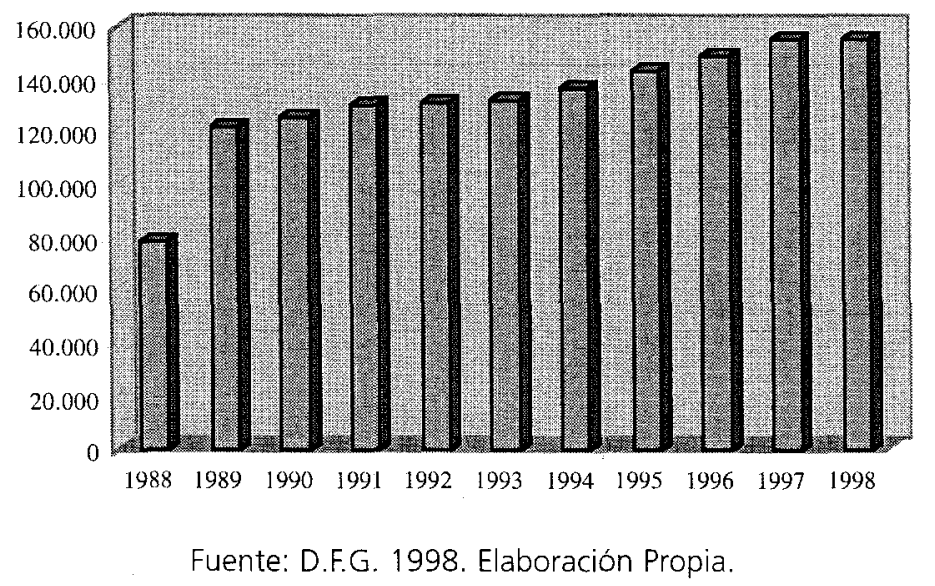

Esta dinámica no es exclusiva de la comarca y también en la provincia se está produciendo el aumento del número de ovejas en unos porcentajes similares -22 y 19\%en el mismo período (1991-1998). A su vez, se están incrementando las cifras de unidades productivas que presenta este tipo de ganado, con medias por explotación próximas a las de nuestra comarca, 64 frente a 53 en Donostialdea-Bidasoa (Campaña de Saneamiento del ganado ovino. D.F.G., 1999). La media de la Comunidad Autónoma Vasca, aun siendo más elevada (71 ovejas/rebaño), indica una situación equiparable, con el predominio de una cabaña reducida, muy alejada de las reses necesarias para completar una U.T.A.

Sin embargo, si en el caso del vacuno el paso de una explotación intensiva a una extensiva era un hecho evidente, señalado por los propios ganaderos que se acogen 
a los planes de abandono y coincidente en el número de cabezas y explotaciones, en el lanar esta realidad no está tan contrastada?. Trataremos a continuación de profundizar en el papel del ovino en este proceso de sustitución.

\subsection{La tipología de explotación residual, la única singularizada}

Procedemos a clasificar y cuantificar las explotaciones recogidas en la Campaña de Saneamiento de ovino referida a 1998 de todos los municipios de la comarca, en función de su grado de dedicación a esta actividad.

Tabla 3. U.T.A. en explotaciones de ovino. Año 1998.

\begin{tabular}{|c|c|c|}
\hline MUNICIPIO & $>1$ U.T.A. & $<1$ U.T.A. \\
\hline ANDOAIN & 5 & 42 \\
\hline ASTIGARRAGA & 0 & 17 \\
\hline DONOSTIA & 6 & 34 \\
\hline ERRENDERIA & 2 & 24 \\
\hline HERNANI & 8 & 55 \\
\hline HONDARRIBIA & 4 & 12 \\
\hline IRUN & 5 & 68 \\
\hline LASARTE-ORIA & 1 & 9 \\
\hline LEZO & 2 & 8 \\
\hline OIARTZUN & 14 & 105 \\
\hline PASAIA & 2 & 2 \\
\hline URNIETA & 1 & 39 \\
\hline USURBIL & 1 & 24 \\
\hline$\overline{\text { COMARCA }}$ & 51 & 439 \\
\hline
\end{tabular}

Fuente: D.F.G. 1998. Elaboración Propia.

El $90 \%$ de las explotaciones que poseen ganado ovino no alcanzan la U.T.A. en esta actividad. Tan sólo 51 rebaños justifican al menos una persona dedicada a tiempo completo. En el ámbito municipal, Oiartzun es el término que tiene un mayor número de hatos tanto en uno como en otro grupo y junto a él, Hernani y Donostia. Otros municipios prácticamente carecen de este tipo de actividad (Usurbil, Urnieta, LasarteOria y Astigarraga).

El análisis de las distintas tipologías de explotación diferenciadas lo efectuamos mediante el empleo de los Registros, tomando como referencia explotaciones recogidas en la Campaña de Saneamiento. En este caso, hemos optado por elegirlas del municipio de Oiartzun puesto que concentra el mayor número de explotaciones profesionales y marginales, cuenta con una asociación de ganaderos que gestiona los montes de utilidad pública y es el único que nos aporta una visión de conjunto de la situación de este sector en la comarca. 
Tabla 4. Muestra de explotaciones de ovino no especializadas. Año 1999.

\begin{tabular}{|l|c|c|c|c|c|c|c|c|c|c|c|c|}
\hline MuESTRA & $N^{\circ}$ C. & Compl. & Edad & Dedic. & Cóny. & Otras & \multicolumn{2}{|c|}{ Res. Cultivos (Ha) } & \multicolumn{3}{|c|}{ Rég. Tenencia (Ha) } \\
\cline { 5 - 11 } MuNICIPIO & Ovino & Bov. & Titular & T/P & Ayuda & Ayuda & Forraj. & Forest. & Otros & Propied & Arrend. & Otros \\
\hline Muestra 1 & 51 & & 29 & $\mathrm{P}$ & & & 6,4 & 7,2 & 0,4 & 6,9 & & 7,0 \\
Muestra 2 & 28 & 28 & 71 & $\mathrm{P}$ & $\mathrm{T}$ & $\mathrm{P}$ & 5,6 & 5,3 & 0,4 & 10,3 & 1,0 & \\
Muestra 3 & 27 & & 48 & $\mathrm{P}$ & & & 11,5 & & & & & 11,5 \\
Muestra 4 & 71 & & 35 & $\mathrm{P}$ & & & 6,2 & 5,7 & 0,5 & 11,4 & & 0,9 \\
Muestra 5 & 17 & & 44 & $\mathrm{P}$ & & & 0,7 & & 0,3 & 1,0 & & \\
Muestra 6 & 24 & & 64 & $\mathrm{P}$ & & & 0,7 & & & 0,7 & & \\
Muestra 7 & 87 & 1 & 67 & $\mathrm{~T}$ & & & 30,3 & & & 11,7 & & 18,6 \\
Muestra 8 & 117 & 8 & 58 & $\mathrm{~T}$ & & & 52,7 & & & 5,3 & 3,7 & 43,7 \\
Muestra 9 & 74 & & 27 & $\mathrm{P}$ & & & 3,0 & & & 3,0 & & \\
Muestra 10 & 11 & & 73 & $\mathrm{~T}$ & $\mathrm{P}$ & & 4,4 & 0,3 & 0,2 & 5,0 & & \\
Muestra 11 & 86 & & 34 & $\mathrm{P}$ & & $\mathrm{T}$ & 11,9 & & 0,4 & & 3,2 & 9,1 \\
Muestra 12 & 70 & 28 & 55 & $\mathrm{~T}$ & & $\mathrm{~T}$ & 13,9 & 16,7 & 1,5 & 4,4 & 7,2 & 20,6 \\
Muestra 13 & 112 & 7 & 58 & $\mathrm{~T}$ & $\mathrm{~T}$ & & 17,6 & 0,5 & 1,6 & 3,2 & 8,5 & 7,9 \\
\hline Total & 775 & 72 & 663 & 9,0 & 2,5 & 2,5 & 164,9 & 35,7 & 5,3 & 62,9 & 23,7 & 119,3 \\
\hline Expl. Media & 59,6 & 5,5 & 51 & 0,7 & 0,2 & 0,2 & 12,7 & 2,7 & 0,4 & 4,8 & 1,8 & 9,2 \\
\hline
\end{tabular}

Fuente: Registro de Explotaciones. D.F.G. 1999. Elaboración Propia.

Si observamos la relación entre las actividades reflejadas y el grado de dedicación y ayuda en las unidades representadas, podemos diferenciar situaciones distintas dentro de esta muestra.

- En dos casos, se trata de activos con dedicación exclusiva, que combina una cabaña ovina considerable con otra actividad ganadera, normalmente vacuno de leche. Constituyen explotaciones que rondan una U.T.A. pero con jefes de explotación de edades próximas a los 60 años y que carecen de relevo en la unidad familiar. Probablemente hayan iniciado el proceso de abandono de la actividad.

- En otros tantos, la ayuda del hijo, aun siendo a tiempo parcial, se evidencia en la considerable carga ganadera que tienen. Sin embargo, la actividad agrícola principal no es ovina sino bovina y en este caso de abasto.

- En el resto de los ejemplos (9), sea titular mayor de 65 años o joven, se mantiene un pequeño rebaño, a menudo inferior a 30 cabezas, sin ningún otro complemento, con una dedicación parcial de toda la población en edad activa.

Otra de las particularidades la presenta la tenencia del suelo utilizado, siendo el principal el arrendado o recogido en otros regímenes $(9,2 \mathrm{Ha})$, superior a la superficie en propiedad $(4,8 \mathrm{Ha})$. El disfrute de comunales explicaría esta situación. Son utilizados especialmente por las explotaciones que mayor cabaña pecuaria ostentan aunque también a este grupo se le unen algunas que apenas poseen ganado. La declaración del terreno utilizado en estos comunales se ha de tomar a modo indicativo dado que a menudo el rebaño deambula en estado semilibre en un área o zona de la que se declara una superficie aproximada ${ }^{8}$. 
En el mapa $n^{\circ} 2$ cartografiamos, a partir de las referencias del registro y de su contrastación con las del catastro de rústica, los usos del suelo de una explotación que estuvo especializada en ovino pero que, dada la edad del titular y la falta de continuidad, actualmente apenas mantiene un reducido rebaño y ya ha dejado de utilizar los comunales que hasta hace bien poco aprovechaba. Como elemento más destacado cabe reseñar la ubicación que habitualmente presenta el caserio ovino, normalmente en zonas más altas y con más pendientes, sobre las que dominan los usos forrajeros, aunque entre éstos suele ser el pastizal el más representado.

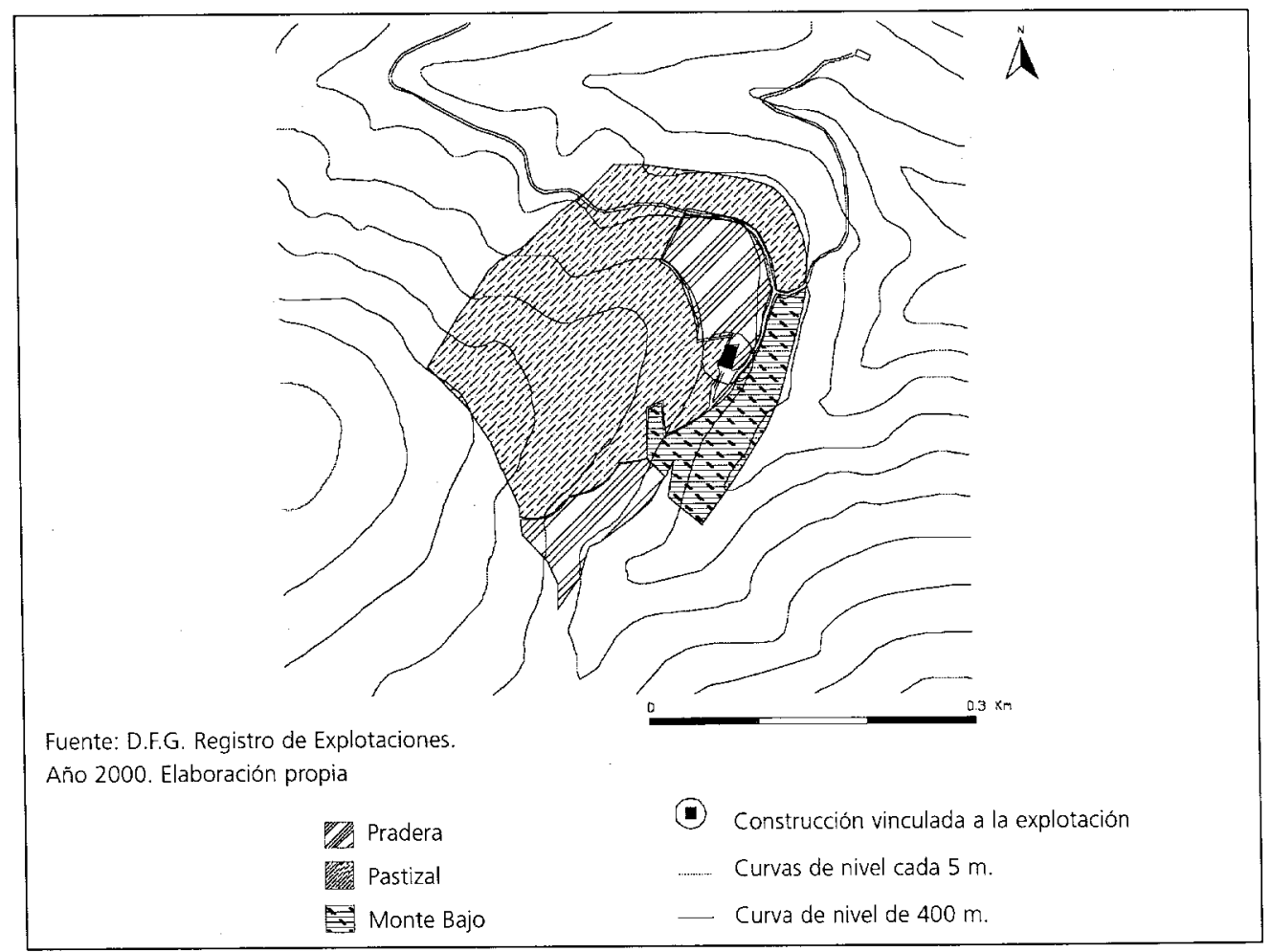

Figura 3. Explotación especializada en ovino con menos de una UTA (Olartzun). Año 2000.

Las explotaciones de ovino especializadas que justifican más de una U.T.A., en función de la muestra tomada en el municipio de Oiartzun, mostrarían unas características sensiblemente distintas.

El tamaño medio de los rebaños, 214 ovejas, justifica al menos una persona con dedicación exclusiva a la actividad. A menudo la combinan con bóvidos, tanto de leche como de carne. El caserío aprovecha todas sus posibilidades, desde el comunal hasta la pradera. 
Tabla 5. Muestra de explotaciones de ovino especializadas. Año 1999.

\begin{tabular}{|l|c|c|c|c|c|c|c|c|c|c|c|c|}
\hline MUESTRA & $N^{\circ}$ C. & Compl. & Edad & Dedic. & Cóny. & Otras & \multicolumn{2}{|c|}{ Res. Cultivos (Ha) } & \multicolumn{2}{|c|}{ Rég. Tenencia (Ha) } \\
\cline { 7 - 12 } MUnicipio & Ovino & Bov. & Titular & $\mathrm{T} / \mathrm{P}$ & Ayuda & Ayuda & Forraj. & Forest. & Otros & Propied & Arrend. & Otros \\
\hline Muestra 1 & 218 & 1 & 65 & $\mathrm{~T}$ & $\mathrm{P}$ & & 44,4 & & 1,1 & 3,2 & 3,2 & 39,0 \\
Muestra 2 & 213 & 7 & 63 & $\mathrm{~T}$ & & $\mathrm{~T}$ & 28,1 & 1,6 & 1,0 & 9,8 & & 20,9 \\
Muestra 3 & 185 & 10 & 63 & $\mathrm{~T}$ & $\mathrm{~T}$ & & 40,0 & & 0,3 & 5,4 & 4,6 & 30,3 \\
Muestra 4 & 359 & & 33 & $\mathrm{~T}$ & & & 51,5 & 1,0 & & 18,6 & & 33,8 \\
Muestra 5 & 195 & 16 & 88 & $\mathrm{P}$ & $\mathrm{T}$ & $\mathrm{T}$ & 34,5 & 1,0 & 0,4 & 8,5 & 7,5 & 19,9 \\
Muestra 6 & 216 & 4 & 33 & $\mathrm{~T}$ & & & 2,4 & & & & & 2,4 \\
Muestra 7 & 165 & 9 & 51 & $\mathrm{P}$ & $\mathrm{T}$ & $\mathrm{T}$ & 28,6 & & 0,2 & 10,1 & 2,3 & 16,3 \\
Muestra 8 & 160 & 18 & 65 & $\mathrm{~T}$ & & & 15,8 & 0,6 & & 15,1 & 1,2 & \\
Muestra 9 & 260 & & 63 & $\mathrm{P}$ & $\mathrm{P}$ & & 40,5 & 0,3 & 0,5 & 1,0 & 12,1 & 28,3 \\
Muestra 10 & 185 & & 29 & $\mathrm{~T}$ & & $\mathrm{P}$ & 40,4 & & & 0,2 & 3,8 & 36,4 \\
Muestra 11 & 206 & & 58 & $\mathrm{~T}$ & & & 82,5 & & & & 41,9 & 40,6 \\
\hline Total & 2.362 & 65 & 611 & 9,0 & 2,5 & 2,5 & 408,8 & 4,4 & 3,6 & 72,0 & 76,7 & 268,0 \\
\hline Expl. Media & 214,7 & 5,9 & 56 & 0,8 & 0,2 & 0,2 & 37,2 & 0,4 & 0,3 & 6,5 & 7,0 & 24,4 \\
\hline
\end{tabular}

Fuente: Registro de Explotaciones. D.F.G. 1999. Elaboración Propia.

El régimen de tenencia en propiedad presenta similitudes con el grupo no especializado pero no el espacio utilizado. El aprovechamiento forrajero es el dominante $(37,2 \mathrm{Ha})$ y es el mayor disfrute de comunales el que aporta las diferencias entre la base utilizada de uno y otro grupo ya que la superficie en propiedad es similar en ambos casos. Las parcelas en arrendamiento o en otros usos superan las 31 Ha mientras que la superficie en propiedad es de 6,5 Ha.

El análisis de la dedicación, la ayuda familiar, edad y número de cabezas nos aporta una doble diferenciación dentro de este grupo:

- Por un lado tenemos una tipología de caseríos con jefes de explotación de edades superiores a 50 años que carece de continuidad y que, a pesar de mantener una cabaña todavía importante, es previsible que vayan reduciendo progresivamente su actividad ( 5 de las 11 muestras se encontrarían en esta situación).

- Por otro, estaría aquella tipología formada por caseríos que tienen continuidad. No muestran diferencias significativas respecto al anterior grupo en cuanto a actividad, si bien es previsible que en un futuro inmediato la presenten.

Aunque no se deduce de esta información, la estrategia de unos y otros es claramente distinta. Mientras en el primer grupo, el de la población de edad más avanzada, el destino final de la producción es la venta directa de leche o la venta a la central, en el segundo se están consolidando progresivamente algunos proyectos de transformación de leche en derivados, sobre todo queso ${ }^{9}$, tratando de obtener el mayor valor añadido posible a la producción de leche de oveja. 


\subsection{Una actividad marginal}

El ganado lanar ha estado tradicionalmente presente en el caserio de la comarca, aunque generalmente como actividad secundaria y marginal. En un momento en el que se produce el abandono del vacuno de leche, el ovino le sustituye y se convierte a menudo en la única actividad ganadera de la explotación pero sin aumentar su anterior importancia. Estos rebaños los conforman unas decenas de reses cuyo único aprovechamiento se reduce a su disfrute cárnico mediante la retirada del cordero lechal.

Llama la atención el aumento del número de explotaciones y de la cabaña ovina en la última década, si bien se mantienen en unas medias similares, próximas a 50 cabezas por rebaño. Dos razones justifican esta situación. Por un lado, al retirar el ganado vacuno el ganadero decide conservar o recuperar un pequeño rebaño de ovejas que existía en el caserío, y por otro, con el objeto de cobrar la prima de ovino, muchos hatos que a menudo no eran declarados se oficializan ahora y acceden a los derechos de ayuda ${ }^{10}$.

La profesionalización del sector y especialización en esta actividad es escasa y apenas alcanza en la comarca una veintena de explotaciones. Es más, el análisis de elementos que marcan su dinamismo y que doblan los beneficios por litro de producción, como es la transformación de la leche de oveja en derivados, principalmente queso y cuajada, reducen este grupo a menos de la mitad. En este momento, en la comarca no se supera la decena de explotaciones que cuenten con una estructura mínimamente acondicionada para la elaboración de derivados de leche de oveja ${ }^{11}$.

Se vuelve a repetir una situación habitual en la mayoría de los sectores productivos, marcada por una dualidad entre el abandono y la especializacion. En este caso está representada por un grupo mayoritario en el que el ovino es una actividad marginal, otro en el que ésta se está reduciendo progresivamente, preferentemente por la avanzada edad de los jefes de explotación y un tercero, inferior al 5\% de los rebaños, que ha iniciado una estrategia tendente a obtener un mayor valor añadido de su producción en una dinámica de especialización y profesionalización.

\section{Los registros y los usos del suelo: un aprovechamiento forrajero}

El carácter individualizado del registro de explotaciones dificulta realizar un estudio masivo de unidades agrarias. En la mayoría de los casos hemos de recurrir al análisis de un reducido número de muestras y extrapolar los resultados a una realidad más amplia. En la investigación de los usos del suelo volvemos a encontrarnos con esta problemática, que nos lleva a elegir un barrio rural como muestra, los suficien- 
temente reducido para que el investigador pueda contrastar los datos que han sido recogidos en los registros. En el ejemplo que estamos analizando tomamos como referencia el barrio de Okendoene, en Hernani. Esta área, con un marcado carácter rural, esta constituida por una decena de explotaciones, con algunos de los suelos de mejores condiciones agrológicas que se conservan en todo el valle.

\subsection{La heredad no condiciona la continuidad de la explotación}

Al igual que en el análisis de las distintas explotaciones de ovino, recogemos a continuación los caracteres más significativos de las unidades agrarias del barrio de Okendoene, siguiendo la información de los registros. A diferencia del anterior epígrafe, no hacemos una selección previa y tomamos como referencia todas las explotaciones de la zona.

Tabla 7. Explotaciones agrarias. Barrio Okendoene (Hernani). Año 2001.

\begin{tabular}{|c|c|c|c|c|c|c|c|c|c|c|c|c|}
\hline Muestra & \multicolumn{2}{|c|}{$N^{\circ}$ Reses } & Edad & Dedic. & Cóny. & Otras & \multicolumn{3}{|c|}{ Res. Cultivos ( $\mathrm{Ha}$ ) } & \multicolumn{3}{|c|}{ Rég. Tenencia ( $\mathrm{Ha})$} \\
\hline Okendoene & Fris. & Carne & Titular & $\mathrm{T} / \mathrm{P}$ & Ayud. & Ayud. & Forraj & Forest. & Otros & Prop. & Arrend & Otros \\
\hline Muestra 1 & 2 & 6 & 51 & $\mathrm{P}$ & $\mathrm{T}$ & & 6,3 & 2,7 & 0,4 & 9,3 & & \\
\hline Muestra 2 & & 3 & 56 & $\mathrm{~T}$ & $\mathrm{P}$ & & 18,3 & 3,4 & 0,8 & 19,8 & & \\
\hline Muestra 3 & & 33 & 40 & $\mathrm{~T}$ & & $\mathrm{~T}$ & 14,9 & 3,0 & 3,6 & 21,3 & & \\
\hline Muestra 4 & & 36 & 51 & $\mathrm{~T}$ & & $\mathrm{p}$ & 18,2 & 3,0 & 4,4 & 18,2 & 0,6 & 6,8 \\
\hline Muestra 5 & 2 & 2 & 31 & $\mathrm{~T}$ & & $T(2)$ & 4,5 & 5,2 & 1,5 & 10,9 & 0,2 & \\
\hline Muestra 6 & 8 & & 57 & $\mathrm{~T}$ & & $\mathrm{P}$ & 4,9 & 5,0 & 3,9 & 13,3 & & \\
\hline Muestra 7 & 3 & & 51 & $\mathrm{P}$ & & & 0,9 & 4,0 & 0,3 & 5,2 & & \\
\hline Muestra 8 & 48 & & 49 & $\mathrm{~T}$ & & & 15,5 & & 1,1 & 9,0 & 2,7 & 4,9 \\
\hline Muestra 9 & 1 & 6 & 76 & $\mathrm{P}$ & & $\mathrm{P}$ & 8,4 & & 0,8 & 7,4 & 1,3 & \\
\hline Muestra 10 & & 15 & 51 & $\mathrm{~T}$ & $\underline{P}$ & & 11,7 & 4,5 & & 15,2 & & \\
\hline Total & 64 & 101 & 513 & 8 & 2 & 5,5 & 103,5 & 30,7 & 16,9 & 130 & 4,8 & 11,7 \\
\hline Explo. Media & 6,4 & 10,1 & 51,3 & $\overline{0,8}$ & 0,2 & 0,6 & 10,3 & 3,1 & 1,7 & 13,0 & 0,5 & 1,2 \\
\hline
\end{tabular}

Fuente: Registro de Explotaciones. D.F.G. 2001. Elaboración Propia.

El primer aspecto que llama la atención de los resultados obtenidos es que estamos ante un caserio con un dominio sensiblemente más amplio al que hemos recogido en las muestras anteriores, con una SAU media de alrededor de $10 \mathrm{Ha}$. La inexistencia de nuevas construcciones, el mantenimiento del mayorazgo y la disponibilidad de unos suelos con buenas condiciones agrológicas ha posibilitado la consecución de una unidad más completa. El carácter ganadero de este caserío queda reflejado en el aprovechamiento que confiere a su heredad y a la que arrienda, que destina casi exclusivamente a la obtención de hierba para forraje, alrededor de 10 de las $15 \mathrm{Ha}$ que utiliza. 
Por otro lado, se observa que la mayoría de las explotaciones ostentan una orientación bovina. Tan sólo la muestra n ${ }^{\circ} 5$ presentaría una orientación hortícola, tres más tendrían una orientación láctea y las seis restantes estarían especializadas en ganado de carne. Otras actividades, como el ovino o la manzana de sidra, también están representadas pero su importancia económica es muy reducida. Entre los usos forestales destacan las repoblaciones de coníferas, pino insignis y en un caso laricio, aunque las parcelas destinadas son en todos los ejemplos de pequeña dimensión, nunca superiores a las $2 \mathrm{Ha}$.

El régimen de tenencia en propiedad es el más representado. Las parcelas arrendadas o cedidas no llegan a suponer el $10 \%$ del total del suelo utilizado, concentradas en su mayoría en dos unidades productivas. El aprovechamiento prioritario de éstas es el de pradera si bien, en un caso, coincidente con la muestra $n^{\circ} 4$, la mayoría de las parcelas utilizadas están ocupadas por monte bajo y frondosas $(4.75 \mathrm{Ha})$ y pastizal $(1,2 \mathrm{Ha})$.

La relación de los distintos factores que se analizan en cada explotación aporta a su vez una serie de conclusiones a tomar en consideración:

- Observamos que de las 9 explotaciones orientadas en ganadería 6 tienen una actividad inferior a una UTA. Coinciden con titulares de edad avanzada, con población de dedicación parcial o, como en el caso de las muestras $n^{\circ} 1,2$ y 10 , con mujeres que aunque presentan una dedicación exclusiva realmente están empleadas en otras labores ${ }^{12}$. Aunque hay alguna excepción, utilizan sólo terrenos de su propiedad.

- En dos muestras, en ambos casos orientadas en vacuno de carne, se llega a superar la UTA. Corresponde a población en edad activa que combina esta actividad con otra catalogada como agricola, como es la elaboración y degustación de sidra. En un caso utilizan terrenos arrendados o cedidos, aunque sensiblemente inferiores a los que tienen en propiedad y normalmente más marginales, con un domino del pastizal.

- Tan sólo la muestra $n^{\circ} 8$, especializada en vacuno de leche, supera la segunda UTA. Coincide con población inferior a cincuenta años al frente de la explotación, de dedicación exclusiva y que utiliza una considerable superficie en arrendamiento o cesión. El aprovechamiento prioritario es la pradera, con alguna pequeña parcela destinada a cultivos forrajeros $(1,1 \mathrm{Ha})$.

Los registros de explotaciones, por medio de la identificación del uso y la parcela en el catastro de rústica, permiten definir los aprovechamientos que se le aporta al suelo rural de una determinada área, quién se lo aporta y el tipo de convenio que se establece entre el propietario y el usuario. Apoyándonos en la entrevista descubrimos, a su vez, la distinta intensidad de aprovechamiento que se le da a cada parcela. 
Del análisis de las explotaciones del barrio Okendoene de Hernani, y en función de los usos y regímenes del suelo cabe resaltar en primer lugar que son muy pocos los ganaderos que utilizan terrenos de otros. Tan sólo la explotación especializada en vacuno de leche maneja parcelas de otros ganaderos, en este caso de al menos tres vaquerias distintas.

La entrevista nos permite profundizar en la distinta intensidad que se le aporta al aprovechamiento dependiendo del régimen de tenencia. Nuevamente es la ganadería lechera la que mejor representa esta afirmación, que llega a obtener cuatro siegas del terreno que disfruta en propiedad, mientras las ganaderías especializadas en carne le aportan uno o dos cortes para luego dejar pastar al ganado en la parcela.

El registro de explotaciones presenta una serie de lagunas a hora de recoger su información, fácilmente subsanables recurriendo la entrevista, por ejemplo, si queremos descubrir la intensidad del aprovechamiento real de un terreno. Del mismo modo, observamos cómo el propietario del terreno declara el suelo que arrienda o cede a terceros como si fueran utilizados por él, sin admitir que es otra persona la que realmente trabaja esas parcelas. Tenemos que tener en cuenta que todos los contratos de arrendamiento que se establecen son orales, sin ningún compromiso por parte del propietario ${ }^{13}$. Ello lleva a que, a menudo, en los registros coinciden más de una explotación utilizando la misma parcela, aunque es relativamente sencillo discernir quién es el que realmente la utiliza.

\section{Una fuente novedosa y fundamental en los estudios agrarios}

Los registros que hemos analizado nos muestran algunos de los caracteres principales de la situación, evolución y dinamismo de las explotaciones agroganaderas vascas. Su simple combinación con otras fuentes de información o su sencilla corroboración recurriendo a entrevistas seleccionadas convierte el uso de esta fuente en obligatorio en el estudio de las unidades agrarias. Entre otras, recogemos a continuación algunas de las conclusiones que hemos obtenido a través de la utilización de estos registros.

En primer lugar, observamos en el conjunto de labores estudiadas dos tendencias claramente identificadas entre las explotaciones que hemos analizado. Una, que asegura el mantenimiento de una ocupación agrícola de cierto peso a medio-largo plazo y otra, en la que esta actividad va claramente disminuyendo, situándose en unos rendimientos cuasi marginales. Existe también una tercera, en una posición intermedia, que no presenta ni una actividad considerable ni una dinámica regresiva ${ }^{14}$. 
En el primer tipo, el que muestra un peso específico y una continuidad a medio plazo garantizada, la asignación a la actividad es exclusiva. Se apuesta por un modelo de instalación especializada, con una estrategia dirigida al aumento de la capacidad de rendimiento, una intensificación de los métodos de producción y una capitalización cada vez mayor ${ }^{15}$. En esta agrupación no están incluidas ni todas las instalaciones que superan la U.T.A. ni siquiera todas las que forman parte de asociaciones profesionales, pero sí la mayoría de las que integran estas últimas.

El segundo grupo, el que actualmente muestra menos de una unidad de trabajo agrícola, es el más generalizado en todos los sectores de producción. Lo componen caseríos con jefes mayores de 60 años y que carecen de continuidad al frente de la explotación. Junto a ellos, otro subgrupo estaría formado por titulares con edades inferiores pero que ejercen la actividad agraria a tiempo parcial y cuyos ingresos proceden en su mayoría de fuera de la explotación.

La dinámica de este conjunto es claramente regresiva o, en todo caso, dedicada al mantenimiento de una actividad marginal. En algunos ejemplos sólo perdurarán labores de segundo orden, que siempre han presentado un valor económico marginal, como el ovino. En otros, marcan el paso de actividades intensivas a extensivas, disminuyendo o el número de cabezas o el número de hectáreas utilizadas. En esta situación se encontrarían al menos 8 de cada 10 explotaciones en la comarca.

Un último grupo estaría compuesto por explotaciones difíciles de encajar en alguna de las otras dos tipologías. Normalmente muestran una dedicación superior a una U.T.A. e inferior a dos U.T.A. En él agrupamos a aquellas instalaciones que presentan un estado estable, que no aumenta ni disminuye su capacidad productiva, para las que la actividad agraria no constituye su principal fuente de ingresos. Nos referimos, entre otras, al bovino de carne, en el que dominan unidades que ligan agricultura a una fuente de ingresos exterior.

El factor que principalmente condiciona la decisión de proseguir con una función agrícola mínima es la edad del jefe de la explotación. Sea cual sea la actividad, toda población mayor de 55 años que carece de ayuda familiar joven en la explotación forma parte del grupo en el que la agricultura está en proceso regresivo o se encuentra ya en un estado marginal. Esta situación es extensible a aquellos jefes de explotación de edad incluso inferior que, presentando ocupación parcial o exclusiva y una labor superior a una U.T.A., carecen también de continuidad.

En el caso de los titulares menores de 55 años, es el grado de dedicación a la actividad (exclusiva/parcial) el que marca la diferencia sustancial entre las explotaciones. En términos generales, podemos decir que la actividad agrícola aumenta sólo cuando hay población joven al frente o trabajando en la explotación con dedicación 
exclusiva $^{16}$. La asignación parcial del jefe y en gran medida también del joven que ayuda, está ligada a caseríos que tienen actualmente o una ocupación marginal o que simplemente mantienen la actividad.

El caserio, por otro lado muestra una base territorial que ronda entre las 4 y las $12 \mathrm{Ha}$. Es una dimensión homogénea, que muestra un dominio sin grandes diferencias en la mayoría de los casos, independientemente del grado de actividad que presente. Las desigualdades, sin embargo, se establecen en función del grado de dedicación y de la capacidad productiva que tiene la explotación y se evidencia no en la heredad sino en aquellas parcelas que le son arrendadas o cedidas para su uso ${ }^{17}$. Así, mientras unos caseríos tan sólo utilizan terreno en propiedad otros la duplican o triplican a cuenta de suelo arrendado y/o cedido. Son las explotaciones más especializadas, con población joven al frente o trabajando en ellas las que amplian la superficie utilizada.

Por tanto, parece evidente que actualmente no es la heredad del caserío la que condiciona su capacidad productiva e incluso su grado de dedicación a la agricultura sino la apuesta que los miembros jóvenes de la unidad familiar realicen respecto a esta actividad y a su futuro.

La posibilidad de contar con una información que recoge, actualiza y combina datos de los caracteres más relevantes de las unidades agrarias permite llegar a conclusiones como las que estamos aportando. El registro es una fuente nueva, no exenta de problemas, de consulta limitada, incompleta, con aspectos que han de redefinirse y mejorarse pero que, desde su inicio, se convierte en referente de obligada consulta en los estudios de geografía agraria.

\section{Notas}

1 Responde a la publicación en el Boletín Oficial del País Vasco del Decreto 84/1993, de 30 de marzo, por el que se crea y regula el Registro de las Explotaciones Agrarias de la Comunidad Autónoma del País Vasco. El Registro es de complementariedad y actualización obligatoria y es requisito imprescindible para acogerse a cualquier medida de fomento, así como a beneficios fiscales o sociales, tanto en favor de la explotación como de sus titulares. Por tanto, es de suponer que aquellas explotaciones que no lo han cumplimentado es porque no han realizado ningún trámite con la administración y éstas son, a todas luces, las explotaciones que menos dedicación agroganadera presentan.

2 En cada campaña de control del ganado vacuno se ordenan los datos por municipios y por explotación. Los epígrafes que se rellenan son los siguientes: código de la explotación, explotación, nombre y apellidos, D.N.I., reses de carne, reses de leche, reses de abasto. Junto a éstos se recoge un resumen municipal. La campaña de saneamiento de ovino presenta una distribución similar. En este caso, para cada municipio se señalan los siguientes datos: código, titular, explotación, número de reses menores de un año, entre 1-2 años, $>2$ años, machos. Con ellos también se incluye un resumen municipal de todas las explotaciones. 
3 Los comunales de la comarca presentan un período en el que el pastoreo no está permitido, normalmente de noviembre a abril. Aún así, observamos que si la mayoría del ganado es retirado, es habitual que algunas reses de bovino de carne y equino permanezcan en estos pastos todo el año (Behemendi, 2000).

4 Asociaciones profesionales de ganaderos surgidas con el fin de regular el uso y disfrute de los pastos en montes comunales existen en Hondarribia, Pasaia, Irun, Oiartzun, Umieta, Andoain y Usurbit (Behemendi, 2000).

5 La asociación ELE (Asociación de oveja Latxa del País Vasco) engloba a la mayoría de los pastores que ostentan una profesionalización en este sector. Aún así, su número es muy reducido y en Gipuzkoa tan sólo 68 personás integraban esta asociación en 1999 (Documento de trabajo ELE, 1999).

6 Datos obtenidos a partir de la Campaña de Saneamiento realizada por el Departamento de Agricultura y Medio Ambiente de la D.F.G. (1999).

7 Las referencias a este proceso son mucho menores aunque también existen. Calcedo en este sentido señalaba que "En el ovino norteño ... han accedido a la explotación nuevos ganaderos con pocas cabezas, algunos procedentes del sector lechero de vacuno o prejubilados por la crisis industrial, lo que hace que, aun aumentando el censo, descienda el támaño medio por rebaño" (1994: 66).

$8 \mathrm{la}$ declaración de la comarca como zona de agricultura de montaña permite acceder al régimen de indemnizaciones compensatorias de montaña (I.C.M.). Al menos hasta la aprobación de la Agenda 2000 la indemnización se limitaba a 1,4 U.G. por hectárea de superficie forrajera. La utilización de comunales son incluidos dentro de la declaración de superficie correspondiente. En éstas observamos cómo algunos ganaderos señalan la parcela aproximada que utilizan de comunal y que declaran posteriormente. En otros, el ayuntamiento opta por dividir la superficie de pasto comunal entre el número de ganado declarado aportando a cada ganadero una cifra aproximada. La misma declaración es utilizada a la hora de solicitar las primas ganaderas, en este caso sobre una carga ganadera inferior a 2 U.G/Ha y año.

9 Según los informes de gestión técnico-económica de explotaciones de ovino en leche y queso, realizados por Lurgintza para la Campaña 1997-98, un rebaño de 291 ovejas cuya producción se destine a la elaboración de queso aporta un margen bruto anual de 4.703 .257 ptas. La cuenta de explotación correspondiente a una explotación media de 473 ovejas que destina su producción a la venta de leche a un precio medio incluso muy alto (177 ptas./litro), obtiene un margen bruto anual de 4.148 .200 ptas. Por tanto, es necesario duplicar la cabaña ganadera y vender la leche a un precio más elevado del que abona la central (120-130 ptas./litro campaña 1998) para obtener unos ingresos similares.

10 El número de ovejas acogidas a prima ha aumentado en Gipuzkoa de 1993 a 1998 en un 20\%. La prima por res, se mantiene, con altibajos, en torno a las 3980 ptas. en el mismo periodo.

11 En este sector la Directiva 92/46 C.E. sobre las nuevas exigencias a la calidad de la leche en Europa presenta una mayor dificultad de aplicación dado que la venta directa o la elaboración sin ningún control de sanidad es la tónica dominante. Su aplicación aceleraría el proceso de abandono de algunos de los rebaños que superan la U.T.A. de dedicación puesto que apenas hay explotaciones acondicionadas y la composición médica de la leche de oveja requerida por la C.E. tan sólo la cumplía el 11,6\% del total de las explotaciones de ovino en 1996 (ENBA, n 28, 1997).

12 Normalmente suele ser la mujer la que aparece como titular-responsable. Clasificar su trabajo se háce a menudo difícil, en gran medida motivado por la diversidad de situaciones que se producen. A esta situación se refiere el sindicato agrario ENBA que, entre otras, resalta "la inexistencia de una clara delimitación entre el trabajo agrario y el doméstico". Relacionan además su trabajo con las explotaciones de base territorial más pequeña, "decreciendo su importancia con el incremento del tamaño de la explotación" (ENBA, $\mathrm{n}^{\circ}$ 56, 1999: 14).

$13 \mathrm{El}$ área que estamos analizando está influenciada por la proximidad urbana. Ello provoca que naclie quiera adquirir compromisos legales con terceros que puedan evitar una recalificación y posible venta de 
sus terrenos. Sin embargo sí interesa al propietario mantener el uso de su heredad. Prost, en referencia a la agricultura en los espacios periurbanos, llega a la conclusión de que si muchos terrenos mantienen su uso agrícola es más por especulación que por su propio valor económico (1994: 148). Ortega Valcárcel analiza distintos ejemplos de zonas agrícolas periurbanas observando en todas ellas el efecto alcista que provoca en los precios del suelo este tipo de agricultura, a menudo ni siquiera realizada por los propietarios de los terrenos (1988: 94).

14 Tendencias coincidentes con las recogidas en diversas investigaciones publicadas a lo largo de la década de los noventa. Ver Etxezarreta, Cruz, García y Viladomiu. 1994. "La agricultura familiar, ante las nuevas políticas agrarias comunitarias"

15 Este grupo coincide básicamente con el que Mauleon, en el caso de las explotaciones vascas de vacuno de leche denominaba como "familia adulta, exclusiva, con sucesor", y con el de "familia joven exclusiva". Concretamente, analizando el modelo de estrategia que presentan resalta la apuesta de éstas por un "modelo de ganadería intensiva: altas producciones, elevada carga ganadera, fuertes inversiones, depen dencia del exterior, etc," (1998: 264).

16 Las apreciaciones que Mauleon hacia en el caso del sector lechero vasco, en términos generales, son aplicables también al resto de actividades agroganaderas principales del País Vasco. Como señala "El optar por la explotación no ha dependido de que éste cuente con cierta dimensión, sino de que en el mundo familiar se den unas condiciones para conservar un proyecto en grupo: contar con un joven con iniciativa y que les guste las características intrínsecas del trabajo ganadero... " (1998: 262).

17. En Donostialdea-Bidasoa la base territorial utilizada en propiedad sigue siendo un elemento definidor del grado de dedicación de la explotación. Sin embargo, ésta cada día va perdiendo más peso y algunos investigadores comienzan a cuestionar la importancia del espacio utilizado como elemento trascendental. Arnalte señala que cla variable dimensión de las explotaciones pierde valor explicativo cuando éstas dejan de ser verdaderas empresas o unidades productivas autónomas... Más interés tiene el análisis de los procesos de concentración de la capacidad productiva y de la dimensión en los estratos altos de la escala de explotaciones (1997: 526).

\section{Bibliografía y fuentes impresas seleccionadas}

Abad Balboa, C. y Naredo, J.M. (1997): Sobre la "modernización" de la agricultura espanola (1940-1995): de la agricultura tradicional a la capitalización agraria y la dependencia asistencial. En Gómez Benito, C. y González Rodríguez, J. J. eds., Agricultura y sociedad en la España Contemporánea. Madrid. Ministerio de Agricultura, Pesca y Alimentación y Centro de Investigaciones Sociológicas, p. 249-316.

Ardatza (1988...): Publicación quincenal de la Unión de Ganaderos y Agricultores Vascos. Vitoria- Gasteiz.
Arnalte Alegre, E. (1997): Formas de producción y tipos de explotaciones en la agricultura española: viejas y nuevas lineas de diferenciación. En Gómez Benito, C. y González Rodríguez, J. J. eds., Agricultura y sociedaden la España Contemporánea. Madrid. Ministerio de Agricultura, Pesca y Alimentación y Centro de Investigaciones Sociológicas, p. 501-532.

Bel, F. (1990): Hipótesis sobre el funcionamiento de la pluriactividad en una zona de montaña. El caso de los Valles Altos de Saboya. En V.V.A.A. Cambio Rural en Europa. Ministerio de Agricultura, Pesca y Alimentación, p. 275-296. 
Bontron J.C. (Dir.) (1994): Entre ville et campagne. Les espaces de périurbanisation. Paris. Ministère de l'agriculture et de la pêche.

Calcedo Ordonez, V. (1999): La reforma de la PAC y sus efectos en el sector agrario cantábrico. En V.V.A.A., Cambios en los Espacios Rurales Cantábricos tras la integración de España en la UE. Universidad de Cantabria, p. 11-77.

Contreras, J. (1997): Estrategias familiares de producción y reproducción. En V.V.A.A., La Agricultura familiar en España. Estrategias adaptativas y politicas agropecuarias. Universidad de Lleida, p. 17-44.

Delios España, E. (1991): La agricultura en espacios periurbanos: el municipio de Alboraya. Valencia, Generalitat Valenciana. Conselleria d'Agricultura i Pesca.

Enba $(1995 \ldots)$ : Revista quincenal de Información Agricola, Ganadera y forestal de Euskadi. Tolosa (Gipuzkoa).

Etxezarreta Zubizarreta, M. (1997): Trabajo y agricultura. los cambios del sistema de trabajo en una agricultura de transformación. En Gómez Benito, C. y González Rodríguez, J. J. eds., Agricultura y sociedad en la España Contemporánea. Madrid, Ministerio de Agricultura, Pesca y Alimentación y Centro de Investigaciones Sociológicas, p. 533-564.

Etxezarreta, M., Cruz, J., García Morilla, M. y Viladomiu, L. (1995): La agricultura familiar ante las nuevas politicas agrarias comunitarias. Madrid, Ministerio de Agricultura, Pesca y Alimentación.

García Azcarate, T. (1995): El futuro del sector lácteo. Situación y previsiones de mercado en el marco de la Unión Europea. Mundo Ganadero, 5, p. 28-35.

García Azcarate, T. (1997): La reforma de la PAC y la agricultura familiar en España. En
V.V.A.A., La Agricultura familiaren España. Estrategias adaptativas y politicas agropecuarias. Universidad de Lleida, p. 129-134.

García Pascual, F. (1998). La ganaderia en Cataluña. Desarrollo y estructuras del complejo ganadero-industrial. Lleida, Milenio.

Gómez Espin, J.M. y Gil Meseguer, E. (1996): El fracaso de la agricultura a tiempo parcial en las pequeñas explotaciones fruticolas de la región de Murcia. Papeles de Geografía, 2324, p. $147-163$.

González, J.J y Gómez Benito, C. (1997): Clases agrarias, estrategias familiares y mercado de trabajo. En Gómez Benito, C. y González Rodriguez, J. J. eds., Agricultura y sociedad en la España Contemporánea. Madrid, Ministerio de Agricultura, Pesca y Alimentación y Centro de Investigaciones Sociológicas, p. 565-580.

IKT (2000): Diagnóstico del sector lácteo. CAV 2000. Vitoria, Gobierno Vasco. Inédito.

Kimhi, A. y López, R. (1999): A note on farmers retirement and succession considerations: Evidence from a household survey. Journal of agricultural economics, 50, p. 154-162.

M.A.P.A.: Anuario de las producciones agrarias y. Anuario de Estadistica Agraria, años 1990-1997. Madrid.

Massot, A. (1997): El impacto de la reforma de la PAC y los Acuerdos del GATT en la posición competitiva del sistema español de producción animal. En V.V.A.A., La Agricultura familiar en España. Estrategias adaptativas y politicas agropecuarias. Universidad de Lleida, p. 83-128.

Mata Olmo, R. (1997): Paisajes y sistemas agrarios españoles. En Gómez Benito, C. Y González Rodríguez, J. J. eds., Agricultura 
y sociedad en la España Contemporánea. Madrid, Ministerio de Agricultura, Pesca y Alimentación y Centro de Investigaciones Sociológicas, p. 109-172.

Mauleon, J.R. (1998): Estrategias familiares $y$ cambios productivos del caserío vasco. Vitoria, Gobierno Vasco.

Naredo, J.M. (1996): La evolución de la agricultura en España (1940-1990). Servicio de Publicaciones de la Universidad de Granada.

Ramos Leal, F. (1991): Estrategias de la distribución alimentaria: perspectivas para el sector hortícola español. Revista de Estudios Agro-Sociales, 157, p. 153-178.

Sumpsi, J.M. (1994): Competitividad y reforma estructural de la agricultura espa- ñola. En V.V.A.A., Modernización y cambio estructural en la agricultura española. Madrid, Ministerio de Agricultura, Pesca y Alimentación, p. 285-332.

Urbina, J. y Costa, J.D. (1992): Año 2.000: más mercados para menos marcas. HontoFniticultura, 9, p. 24-31.

Van Oort, G. (1994): L'agriculture de loisir, une nouvelle forme d'utilisation de l'espace? Le cas de la frange urbaine de la veille d'Utrecht. Bulletin de L'Association de Géographie Française, 71, p. 135-143.

Vaudois, J. (1994): Les zones maraichéres périurbaines: espaces résiduels ou nouvelles formes d'integration des éspaces agricoles aux stratégies urbaines. Bulletin de L'Association de Géograpbie Française, 71, p. $123-134$. 